\title{
More than Our Enemy: Making Space for the Microbiome in Pharmacy Education
}

Sarah P. Collier, PhD ; Abby J. Weldon, PhD'; Jessica L. Johnson, PharmD, BCPS ${ }^{3}$

${ }^{1}$ Department of Pharmaceutical Sciences, Lipscomb University College of Pharmacy, Nashville, TN

${ }^{2}$ Department of Pharmaceutical Sciences, William Carey University School of Pharmacy, Biloxi, MS

${ }^{3}$ Department of Pharmacy Practice, William Carey University School of Pharmacy, Biloxi, MS

\begin{abstract}
The microbiome is the collection of commensal microorganisms along with their genomes inhabiting the human body. Despite the many known beneficial effects of these microbes on human health, the 2016 ACPE Standards for Doctor of Pharmacy curricula describe Medical Microbiology in Appendix 1 with a pathogen-centered focus. Over the last twenty years, evolving biotechnology has enabled a deeper understanding of the microbiome in the context of both wellness and disease. Retail stores are allocating increasing shelf space to commercial probiotic products, while the approach to PharmD training on the selection and use of these natural care products remains static, creating a disproportionate footprint between PharmD curricula and consumer markets. Looking to the future of patient care, we brief pharmacy educators on the current evidence and invite discussion around a proposed revision to the 2025 ACPE Standards that would add language recognizing the beneficial role of the commensal microbiota and expanding therapeutic applications of microbiome supplementation. We suggest a variety of opportunities within Doctor of Pharmacy curricula as leverage points for including relevant aspects of the microbiome in the training of future pharmacists.
\end{abstract}

Keywords: microbiome, pharmacomicrobiomics, curriculum, and microbiology

\section{Friends + Enemies $=$ Frenemies?}

The Accreditation Council for Pharmacy Education's (ACPE) Standards 2016 requires that candidates for a Doctor of Pharmacy degree study "Medical Microbiology: Structure, function, and properties of microorganisms (bacteria, viruses, parasites, and fungi) responsible for human disease, and rational approaches to their containment or eradication."1 The wording of this ACPE Appendix 1 item fails to acknowledge the well-established beneficial effects of the human microbiota, unfairly demonizing microorganisms and portraying the role of the pharmacist as "The Exterminator." Yet we wonder, isn't there more to microbiology than just the collective list of human pathogens? Are we adequately preparing future pharmacists if we are only tackling microbiology in the context of antimicrobial therapies? Herein, we advocate for broadening the scope of medical microbiology education within pharmacy curricula in a manner that recognizes the positive contributions of the microbiota to human health and wellness. We present a variety of potential touchpoints within typical PharmD curricula that could be leveraged to explore an emerging understanding of the microbiome in the context of pharmaceutical sciences and clinical care.

Corresponding author: Sarah P. Collier, PhD

Department of Pharmaceutical Sciences

Lipscomb University College of Pharmacy

Nashville, TN

Email: sarah.collier@lipscomb.edu
The human body is colonized with commensal, or symbiotic, microorganisms of bacterial, viral, and fungal species in at least equal proportion to the number of human cells in the body. ${ }^{2}$ Together with their genome, commonly referred to as the 'microbiome,' these microorganisms occupy the interfaces between the body and the external environment, such as the skin and the gastrointestinal, respiratory, and genitourinary tracts. Each of these anatomical areas possesses its own regional biome that is influenced by local physical and chemical mediators. Beginning at birth, the depth and diversity of an individual's microbiome is dynamically influenced by lifestyle factors, such as diet, environment, and, according to new scientific investigations, medication exposure. ${ }^{3-6}$

It is well known that the species of microorganisms colonizing the body (collectively referred to as microbiota) play an important role in human health by aiding digestion, synthesizing essential vitamins, providing protection from pathogenic organisms, and influencing the development and regulation of the immune system. ${ }^{2-4}$ Microbiota catabolize and convert plant-based complex carbohydrates into short chain fatty acids that influence satiety, energy balance, and metabolism. In addition to vitamins $B$ and $K$, gut microbiota produce gamma-aminobutyric acid (GABA), tryptophan precursors, serotonin, and catecholamines, suggesting that these organisms may influence neurological processes. ${ }^{7}$ Microbiota also defend against colonization by pathogenic microbes through the production of lactic acid and competition for resources. ${ }^{8}$ 


\section{Ignited by Genomics and Precision Medicine}

Rapidly-expanding access to genetic sequencing technologies and bioinformatics is fueling the field of precision medicine and driving a growing body of evidence connecting the microbiome to human health and disease. ${ }^{9}$ Previously, understanding of the diversity and function of the microbiota was limited to organisms amenable to growth within a laboratory culture environment. Next-generation genetic sequencing technology has allowed for high throughput analysis of an increasing number and diversity of microbiota species at an exponentially decreasing cost, allowing scientists to quickly and easily identify the microbial species present and their relative abundance within an individual's microbiome. Functional data useful for patient care decision-making can then be extrapolated from genomic data, for example, using the presence of antibiotic resistance genes to guide empiric antimicrobial selection. ${ }^{10}$ In response to a rapid expansion in gene sequencing technologies, the number of microbiome-related publications has exponentially increased since the year 2002 (Figure 1). These publications range in scope from descriptions of the species present in various anatomical regions to analysis of connections between microbial diversity and health.

This rapidly-expanding body of literature frames the microbiome in new light. Alterations in the microbiota (i.e., dysbiosis) are increasingly associated with the metabolic and inflammatory pathologies associated with obesity, asthma, inflammatory bowel disease, vaginosis, hepatic encephalopathy, diabetes, and mental illness. ${ }^{11-13}$ If dysbiosis is a cause of disease, then microbial supplementation could perhaps be a treatment. Commercial probiotics have shown some beneficial effects that include the reduction of antibioticassociated diarrhea, rotaviral diarrhea, travelers' diarrhea, and chronic constipation, though the optimal dose and the matching of specific species to specific conditions is unknown. ${ }^{12}$ Increased marketing and use of probiotics is driving considerable interest in prebiotic, synbiotic, and nutritional/food-based approaches to microbiome modification/supplementation among public and scientific communities.

Perhaps the most striking example of microbiome research applied to therapeutics is the development and use of fecal microbiota transplantation (FMT) to regenerate a healthy microbiome. FMT, an investigational new drug, is currently used in the treatment of recurrent Clostridioides difficile infections (rCDI) refractory to standard therapies. ${ }^{14}$ FMT administration has transitioned from delivery by colonoscopy to capsules, which has the potential of expanding the pharmacist's role in managing rCDIs. ${ }^{15}$

Additionally, the diversity and composition of the gut microbiota influences the efficacy of certain medications and therapeutic outcomes. For instance, cancer patients with abundances of certain bacterial phyla display improved therapeutic responses to immune checkpoint blockade immunotherapies, reduced immunotherapy toxicity, and enhanced chemotherapeutic efficacy. Distinct bacterial phyla of the gut microbiome can also reduce graft-versus-host disease following hematopoietic stem cell transplant. ${ }^{16}$ These observations suggest that the interaction between a patient's medications and microbiome (i.e., pharmacomicrobiomics) could be incorporated into therapeutic decision making and thereby add another tool to the field of personalized medicine. ${ }^{16,17}$

\section{Touchpoints across the Pharmacy Curriculum}

Microbiome-related concepts are already being incorporated into teaching and research by some pharmacy educators. Presentations at annual meetings of the American Association of Colleges of Pharmacy (AACP) have described studies on how the gut microbiome influences bioavailability and intestinal metabolism of pharmacotherapeutics, as well as how genetically-modified microorganisms could potentially translate into treatment of enzyme deficiency disorders. ${ }^{18,19}$ Two authors of this commentary (Johnson, Weldon) previously described a microbiome elective course for pharmacy students. ${ }^{20}$ The microbiome and efficacy of probiotic therapies have also served as topics in active learning debate exercises within a pharmacotherapeutics course. ${ }^{14}$

These examples reflect how topics relating to the microbiome are certainly finding a niche audience within pharmacy; however, a greater emphasis is needed for graduates to meet the growing expectations of the field. Studies increasingly suggest that pharmacists seek greater confidence and competence in counseling on complementary and alternative medicines or natural health topics. ${ }^{22-24}$ Patients also recognize probiotic foods and commercial supplements as some of the most commonly used complementary or natural remedies and rely on community pharmacists to provide specific and useful information about them. ${ }^{22-24}$ The evolving landscape of precision medicine research and its application in clinical practice necessitates a perspective shift involving the human microbiota. Although future treatment guidelines and standards of care are anticipated to consider the microbiome in patient care ${ }^{25}$ (similar to how pharmacogenomics is growing to guide therapeutic options), the current required elements of Doctor of Pharmacy curricula fail to encourage student pharmacist exposure to this therapeutic area.

The Accreditation Council for Pharmacy Education's recent announcement of the planned "Standards 2025" offers an ideal opportunity to revise our approach to this topic within pharmacy curricula. The current wording of the ACPE Appendix 1 item "Medical Microbiology" reflects a narrow and biased caricature of microorganisms as the perpetual enemies of humanity. This perspective requires reframing to represent a broader and more inclusive view of the relationships between microbiota and their human hosts that affirms the positive contributions of microbiota to human health. Thus, we propose a modification (shown in bold, italics) of the wording of this 
relevant required element to read "Medical Microbiology: Structure, function, and properties of microorganisms (bacteria, viruses, parasites, and fungi) responsible for human health and disease, and rational approaches to their modification, supplementation, containment or eradication."

This change, if adopted into ACPE Standards 2025, would support and encourage faculty to actively incorporate the microbiome into their classrooms, which is achievable through several different contexts related to pharmacy education (Table 1). Currently, self-care pharmacotherapeutics courses provide general coverage of probiotics, but fail to address the effects that the microbiome may have on the pharmacological efficacy of a drug as seen with anti-cancer therapeutics. Table 1 provides a launchpad for identifying straightforward opportunities to incorporate the microbiome into the PharmD curriculum and clinical experiences. For example, traditional didactic courses like Microbiology or Immunology may include active learning or discussion investigating the role of commensal organisms in immune system development. In the Drug Information or Literature Evaluation classroom, students could undertake the critical examination of the growing body of microbiome literature. In Self-care (Over-the-Counter Medications) or Complementary Medicines (Natural Medicines) courses, students can practice communicating basic science concepts to a public, patient-facing audience seeking counsel on probiotic supplementation. An advanced pharmacy practice experience might include one or more of these suggested ideas into a real-world patient context.

In summary, the growing field of microbiome science advances steadily toward clinical application necessitating a change in health professions education. We invite pharmacy faculty to introduce student pharmacists to the microbiota from a perspective that is more inclusive and nurturing to commensal microbial communities than the historical framework of 'seek and destroy'. In addition to the actions of individual educators, revision of the Medical Microbiology requirement within ACPE Standards 2025 could support continued expansion and adaptation of the role of the pharmacist in response to evolving scientific understanding of the role of the microbiome in human health and disease.

\section{Conflicts of Interest: None Funding/support: None}

The opinions expressed in this paper are those of the author(s).

\section{References}

1. Accreditation Council for Pharmacy Education (ACPE) Accreditation Standard and Guidelines for the Professional Program in Pharmacy Leading to the Doctor of Pharmacy Degree. Accessed August 10, 2021. Available at: https://www.acpeaccredit.org/pdf/Standards2016FINAL.pdf.

2. Sender R, Fuchs S, Milo R. Revised Estimates for the Number of Human and Bacteria Cells in the Body. PLoS Biol. 2016;14(8). doi:10.1371/journal.pbio.1002533

3. Tamburini S, She, N, Wu HC, Clemente, JC. The microbiome in early life: implications for health outcomes. Nature Medicine. 2016;22:713-722. doi:10.1038/nm.4142

4. Singh RK, Chang HW, Yan D, et al. Influence of diet on the gut microbiome and implications for human health. J Trans/ Med. 2017;15 doi:10.1186/s12967-017-1175-y

5. Gimenez-Bastida JA, Carreras LM, Moya-Perez A, Laparra Llopis, JM. Pharmacological Efficacy/Toxicology of Drugs: A Comprehensive Update About the Dynamic Interplay of Microbes. J Pharm Sci. 2018;107:778-84.

6. Vila AV, Collij V, Sanna S, et al. Impact of commonly used drugs on the composition and metabolic function of the gut microbiota. Nat Commun. 2020;11 doi:10.1018/s41467019-14177-z

7. Mayer EA, Tillisch K, Gupta A. Gut/brain axis and the microbiota. J Clin Invest. 2015;125(3):926-938. doi:10.1172/JCI76304

8. Toor D, Wasson MK, Kumar P, et al. Dysbiosis Disrupts Gut Immune Homeostasis and Promotes Gastric Diseases. Int J Mol Sci 2019;10:2432 doi: 10.3390/ijms20102432

9. Proctor LM, Creasy HH, Fettweis JM, et al. The Integrative Human Microbiome Project. Nature. 2019;569(7758). doi:10.1038/s41586-019-1238-8

10. van Schaik W. The human gut resistome. Philos Trans $R$ Soc B Biol Sci. 2015;370(1670). doi:10.1098/rstb.2014.0087

11. Belizario JE, Napolitano M. Human microbiomes and their roles in dysbiosis, common diseases, and novel therapeutic approaches. Front Microbiol. 2015;6:1050 doi.org/10.3389/fmicb.2015.01050

12. Parker EA, Roy T, D'Adamo CR, Wieland LS. Probiotics and gastrointestinal conditions: An overview of evidence from the Cochrane Collaboration. Nutrition. 2018;45:125134.e11. doi:10.1016/j.nut.2017.06.024

13. Foster JA, McVey Neufeld KA. Gut-brain axis: How the microbiome influences anxiety and depression. Trends Neurosci. 2013;36(5). doi:10.1016/j.tins.2013.01.005

14. Geoghegan O, Eades C, Moore LSP, Gilchrist M. Clostridium difficile: Diagnosis and treatment update. Clin Pharm. 2017;9(2). doi:10.1211/CP.2017.20202242

15. Youngster I, Mahabamunuge J, Systrom HK, et al. Oral, frozen fecal microbiota transplant (FMT) capsules for recurrent Clostridium difficile infection. BMC Med. 2016;14:134 doi: 10.1186/s12916-016-0680-9

16. Fessler J, Matson V, Gajewski TF. Exploring the emerging role of the microbiome in cancer immunotherapy. $J$ Immunother Cancer. 2019;7(1). doi:10.1186/s40425-0190574-4

17. Panebianco C, Andriulli A, Pazienza V. Pharmacomicrobiomics: exploiting the drug-microbiota interactions in anticancer therapies. Microbiome. 2018;6(1). doi:10.1186/s40168-018-0483-7 
18. LeBegue CE, Massey JC, Wyatt MD. Phenylalanine Hydroxylase Deficiency: The Pharmabiotic Treatment Approach. Poster. Abstracts Presented at the 120th Annual Meeting of the American Association of Colleges of Pharmacy, Chicago, Illinois, July 13-17, 2019. Am J Pharm Educ. 2019;83(5):7654. doi:10.5688/ajpe7654

19. Dorrestein PC, Ma JD, Momper J, Jarmusch A, Shirley M. SM. TeamScience at Skaggs School of Pharmacy and Pharmaceutical Sciences, UC San Diego. School Poster. 119th Annual Meeting of the American Association of Colleges of Pharmacy, Boston, Massachusetts, July 21-25, 2018. Am J Pharm Educ. 2018;82(5):7160. doi:10.5688/ajpe7160

20. Weldon A, Johnson JL, Mandal M. "Bugs and Biodrugs:" Incorporating Emerging Biological Sciences Topics Into the Pharmacy Curriculum. Oral podium presentation at American Association of Colleges of Pharmacy Virtual Pharmacy Education 2020; July 16, 2020.

21. Viswesh V, Yang H, Gupta V. Evaluation of a modified debate exercise adapted to the pedagogy of team-based learning. Am J Pharm Educ. 2018;82(4):345-353. doi:10.5688/ajpe6278

22. Braun LA, Tiralongo E, Wilkinson JM, et al. Perceptions, use and attitudes of pharmacy customers on complementary medicines and pharmacy practice. BMC Complement Altern Med. 2010;10(1):38-38. doi:10.1186/1472-6882-10-38

23. Kwan D, Boon HS, Hirschkorn K, et al. Exploring consumer and pharmacist views on the professional role of the pharmacist with respect to natural health products: A study of focus groups. BMC Complement Altern Med. 2008;8(1):40-40. doi:10.1186/1472-6882-8-40

24. Dering-Anderson AM. Reflections on 500 call-in radio shows. Am J Pharm Educ. 2018;82(9):1029-1033. doi:10.5688/AJPE7046

25. Dong OM, Howard RM, Church R, et al. Challenges and solutions for future pharmacy practice in the era of precision medicine. Am J Pharm Educ. 2018;82(6):620-626. doi:10.5688/AJPE6652

26. Lee JK, Hume AL, Willis R, et al. Pharmacy competencies for interprofessional integrative health care education. Am J Pharm Educ. 2018;82(6):687-694. doi:10.5688/AJPE6302 
Figure 1. Trends in the number of microbiome publications per year indexed in Pubmed.gov.

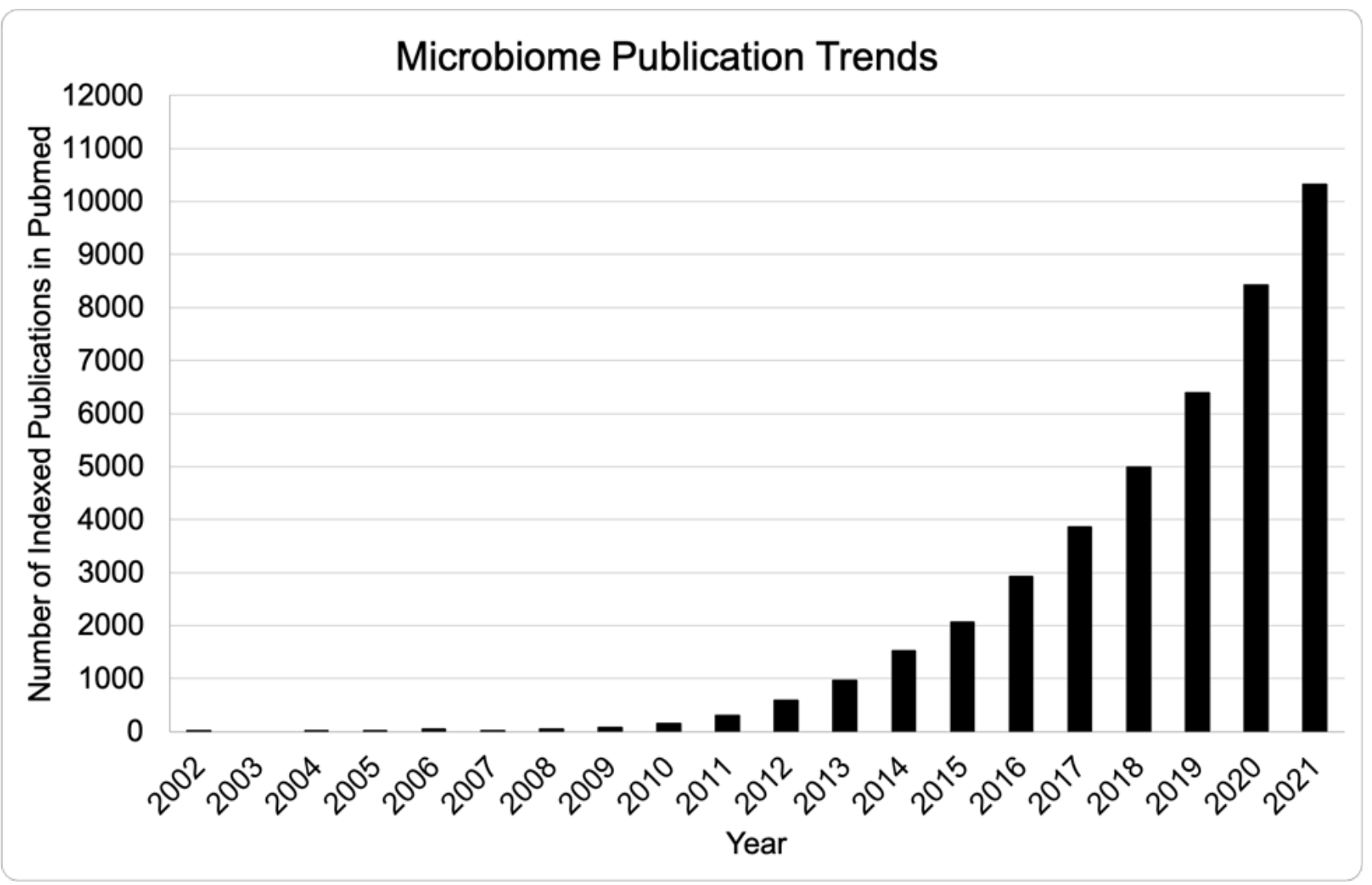

Note: A search was conducted in January of 2022 for all publications with the keyword 'microbiome' in the Title/Abstract field from 2002 to 2021. 
Table 1. Opportunities for Inclusion of Microbiome-Related Concepts into the Doctor of Pharmacy Curriculum

\begin{tabular}{|c|c|}
\hline Curricular Topic Area & Opportunities for Curricular Inclusion of Microbiome-Related Concepts \\
\hline $\begin{array}{l}\text { Pharmaceutics and } \\
\text { Compounding }\end{array}$ & $\begin{array}{l}\text { Compounding requirements for dosage forms containing live microorganisms (packets, } \\
\text { capsules, gummies, topical creams). Storage considerations and product degradation over } \\
\text { time, estimation of shelf life. }\end{array}$ \\
\hline Immunology & $\begin{array}{l}\text { Microbes as an innate defense. Effect of microbial populations on immune effector cells and } \\
\text { on synthesis of immune mediators. }\end{array}$ \\
\hline Biotechnology & Development and use of genetically modified organisms. \\
\hline Anatomy and Physiology & $\begin{array}{l}\text { Emphasis on commensal organisms and their benefit to the human host. Role of the } \\
\text { microbiome in associated organ systems (primarily digestive, respiratory, reproductive, } \\
\text { integumentary). Changes in microbiota across the lifespan. }\end{array}$ \\
\hline $\begin{array}{l}\text { Pharmacokinetics and } \\
\text { Pharmacomicrobiomics }\end{array}$ & $\begin{array}{l}\text { Effects of gut microbiota on drug therapy, including biotransformation and metabolism of } \\
\text { orally administered medications. }\end{array}$ \\
\hline $\begin{array}{l}\text { Self-Care and Patient } \\
\text { Communication }\end{array}$ & $\begin{array}{l}\text { Evaluation of labeling and use of market available pre-and probiotic products. Patient- } \\
\text { focused communication and patient education strategies. }\end{array}$ \\
\hline Pharmacoeconomics & Cost-Benefit of treatment with probiotics to prevent serious disease. \\
\hline Law & Marketing regulations for dietary supplements. \\
\hline $\begin{array}{l}\text { Integrative Health Care, } \\
\text { Complementary and Natural } \\
\text { Medicines }\end{array}$ & $\begin{array}{l}\text { Non-conventional approaches to health including alternative, complementary, and } \\
\text { integrative medicine practices. }{ }^{26}\end{array}$ \\
\hline
\end{tabular}

